Article

\title{
Real-Time and High-Accuracy Arctangent Computation Using CORDIC and Fast Magnitude Estimation
}

\author{
Luca Pilato, Luca Fanucci and Sergio Saponara * \\ Dipartimento Ingegneria della Informazione, Università di Pisa, via G. Caruso 16, 56122 Pisa, Italy; \\ luca.pilato@for.unipi.it (L.P.); luca.fanucci@unipi.it (L.F.) \\ * Correspondence: sergio.saponara@unipi.it; Tel.: +39-050-221-7511; Fax: +39-050-221-7522 \\ Academic Editors: Christos Koulamas and Mihai Lazarescu \\ Received: 11 February 2017; Accepted: 13 March 2017; Published: 16 March 2017
}

\begin{abstract}
This paper presents an improved VLSI (Very Large Scale of Integration) architecture for real-time and high-accuracy computation of trigonometric functions with fixed-point arithmetic, particularly arctangent using CORDIC (Coordinate Rotation Digital Computer) and fast magnitude estimation. The standard CORDIC implementation suffers of a loss of accuracy when the magnitude of the input vector becomes small. Using a fast magnitude estimator before running the standard algorithm, a pre-processing magnification is implemented, shifting the input coordinates by a proper factor. The entire architecture does not use a multiplier, it uses only shift and add primitives as the original CORDIC, and it does not change the data path precision of the CORDIC core. A bit-true case study is presented showing a reduction of the maximum phase error from 414 LSB (angle error of $0.6355 \mathrm{rad)}$ ) to 4 LSB (angle error of $0.0061 \mathrm{rad}$ ), with small overheads of complexity and speed. Implementation of the new architecture in $0.18 \mu \mathrm{m}$ CMOS technology allows for real-time and low-power processing of CORDIC and arctangent, which are key functions in many embedded DSP systems. The proposed macrocell has been verified by integration in a system-on-chip, called SENSASIP (Sensor Application Specific Instruction-set Processor), for position sensor signal processing in automotive measurement applications.
\end{abstract}

Keywords: real-time; Digital Signal Processing (DSP); Embedded Systems; CORDIC (Coordinate Rotation Digital Computer); ASIC (Application Specific Integrated Circuit); FPGA (Field Programmable Gate Array); IP (Intellectual Property); automotive sensors

\section{Introduction}

The CORDIC (Coordinate Rotation Digital Computer) algorithm [1] is used in many embedded signal-processing applications, where data has to be processed with a trigonometric computation. Rotary encoders, positioning systems, kinematics computation, phase trackers, coordinate transformation and rotation are only a few examples [2-4]. One of those is the use of CORDIC as an arctangent computation block, from here referenced as atan-CORDIC. For example, it is at the core of the phase calculation of complex-envelope signals from the in-phase and quadrature components in communication systems, or for angular position measurement in automotive applications, or for phase computation in power systems control and in AC circuit analysis [5-9]. Although the standard CORDIC algorithm is well known in literature, its efficient implementation in terms of computation accuracy and with low overheads in terms of circuit complexity and power consumption is still an open issue [6-16]. To this aim, both full-custom (ASIC in [6]) and semi-custom (IP macrocells synthesizable on FPGA or standard-cell technologies in $[8,9,16]$ ) approaches have been 
followed in the literature. Due to the need for flexibility in modern embedded systems, the design of a synthesizable IP is proposed in this work.

The iterative algorithm of atan-CORDIC, even if implemented in an IPA (Infinite Precision Arithmetic), suffers from approximation errors due to the $n$ finite iteration. Using the ArcTangent Radix (ATR) angles set, and using vectors compliant with the CORDIC convergence theorem [10], the phase error $E_{\theta}$ is bounded by the expression in Equation (1), which depends on the number of iterations $n$. This means that ideally we can reduce the angle computation error using more iterations of the CORDIC algorithm.

$$
E_{\theta} \leq \tan ^{-1}\left(2^{-n+1}\right)
$$

The most accurate way to approximate IPA is by relying on floating-point computation, e.g., using the IEEE 754 double-precision floating-point format. However, when targeting real-time and low-complexity/low-power implementations, the design of fixed-point macrocells is more suited. Moreover, many applications using CORDIC, such as position sensor conditioning on-board vehicles, typically do not adopt floating-point units. For example, in automotive or industrial applications, at maximum 32-bit fixed-point processors are used [5,17].

The implementation of the fixed-point algorithm in a Finite Precision Arithmetic (FPA) implies an accuracy degradation of the phase calculation. The contribution to the errors in FPA is the quantization of the ATR angles set and the quantization on the $x, y$ data path, where rounding or truncation action is taken after the shift operation in each step. Using a similar approach to [11,12], we can estimate a coarse upper-bound of the phase error in FPA, as shown in Equation (2).

$$
E_{\theta} \leq \sin ^{-1}\left(2^{-n+1}+\frac{\varepsilon}{r} \sqrt{2}(n-1)\right)+n \delta
$$

In Equation (2), $n$ is the number of iterations, $\varepsilon$ is the fixed-point precision of the coordinates' data path where the quantization is taken at each loop, $r$ is the magnitude of the starting point and $\delta$ is the angle data path precision. If the initial vector has a magnitude $r$ great enough to neglect the second argument of the $\sin ^{-1}$ function in Equation (2), then the error bound depends mainly on the number of iterations. Due to the quantization in the data paths of the fixed-point implementation, every loop of the algorithm has a truncation error that accumulates itself. This is the meaning of the terms $(n-1)$ and $n \delta$ in Equation (2). Particularly, the latter can lead to an increase of the error upper bound in each iteration. Instead, the $2^{-n+1}$ term indicates the improvement of the error due to more iterations. An example of the dependence of the error upper bound on the number $n$, once the parameters $\varepsilon, r$ and $\delta$ are fixed, will be shown in Section 3. It must be noted that Equation (2) expresses an upper bound to the error, and not the real error (see the difference between the red lines and blue lines in Figures 6-9 in Section 3).

For vectors with a magnitude near zero, the error bound and the estimated error increase ideally up to $\pi / 2$ due the $\sin ^{-1}$ term in Equation (2). The idea proposed in this work is to have a fast estimation of the magnitude of the initial vector and to design a circuit able to magnify the coordinates to let the standard atan-CORDIC work in the area where the phase error is small.

Hereafter, Section 2 presents the fast magnitude estimator. Section 3 discusses the achieved performance in terms of the computation accuracy of the new algorithm. Section 4 discusses the performance of the IP macrocell implementation in CMOS (Complementary Metal Oxide Semiconductor) technology, and its integration in an automotive system-on-chip for sensor signal processing, called SENSASIP (Sensor Application Specific Instruction-set Processor). A comparison of the achieved results to the state-of-the-art in terms of processing accuracy, circuit complexity, power consumption and processing speed is also shown. Conclusions are drawn in Section 5.

\section{Fast Magnitude Estimator and Improved CORDIC Architecture}

The circuit for a fast magnitude estimation is based on the alphamax-betamin technique [13]. The magnitude estimation is a linear combination of the max and min absolute values of the input 
coordinates, as shown in Equation (3). The choice of $a$ and $b$ values depends on the metric that the approximation in Equation (3) wants to minimize. If the max absolute error of the entire input space is the selected metric, the optimum numerical values are $a \approx 0.9551$ and $b \approx 0.4142$. A coarse approximation for a simplified and fast implementation can be $a=1$ and $b=0.5$. This means that only an abs block, a comparison block and a virtual shift plus addition have to be implemented in hardware, while avoiding the use of multipliers. Using the architecture in Figure 1, which implements the calculation in Equation (3), the estimated magnitude $m^{*}$ is always higher than the real one. For example, if the coordinates are equal, i.e., $\left|x_{0}\right|=\left|y_{0}\right|$ the result of Equation (3) is $m *=\left|x_{0}\right| \cdot 3 / 2$ while the real magnitude is $m_{\text {real }}=\left|x_{0}\right| \cdot \sqrt{2}=m * \cdot 2 \sqrt{2} / 3$. In the case of $\left|x_{0}\right|=0$ then $m_{\text {real }}=m *=\left|y_{0}\right|$, while if $\left|y_{0}\right|=0$ then $m_{\text {real }}=m *=\left|x_{0}\right|$. These considerations are shown in Figure 2, where the light grey circumference arc includes the possible positions of the points with the estimated magnitude $m^{*}$. Figure 2 also shows the real position corresponding to the case $\left|x_{0}\right|=\left|y_{0}\right|$ with a black point and two segments, drawn with a black line, connecting this point to the points corresponding to the cases of $m_{\text {real }}=m *=\left|x_{0}\right|$ or $\left|y_{0}\right|$. In Figure 2 the two segments are always under the computed magnitude arc. It should be noted that for all the input points belonging to the two segments, the corresponding magnitude $m^{*}$, estimated with Equation (3) and the circuit in Figure 1, is the radius of the light grey circumference arc in Figure 2.

$$
m *=\operatorname{amax}\left(\left|x_{0}\right|,\left|y_{0}\right|\right)+b \min \left(\left|x_{0}\right|,\left|y_{0}\right|\right)
$$

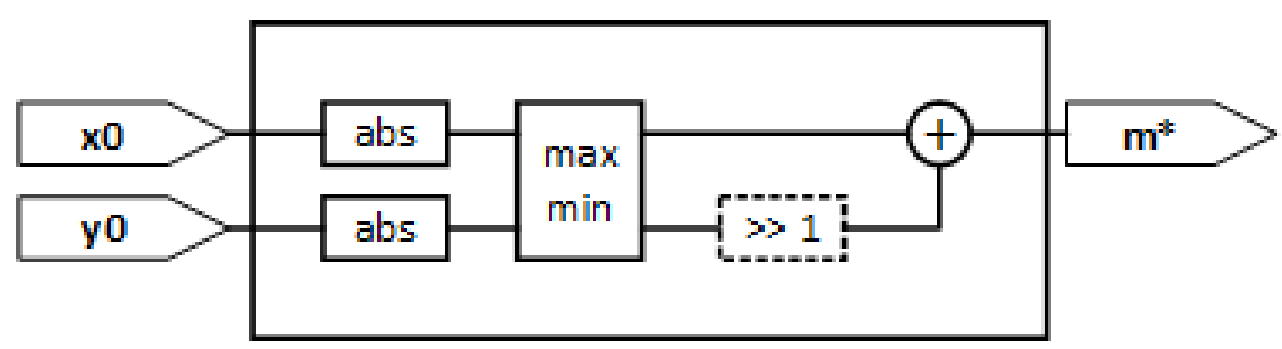

Figure 1. The fast magnitude estimator architecture.

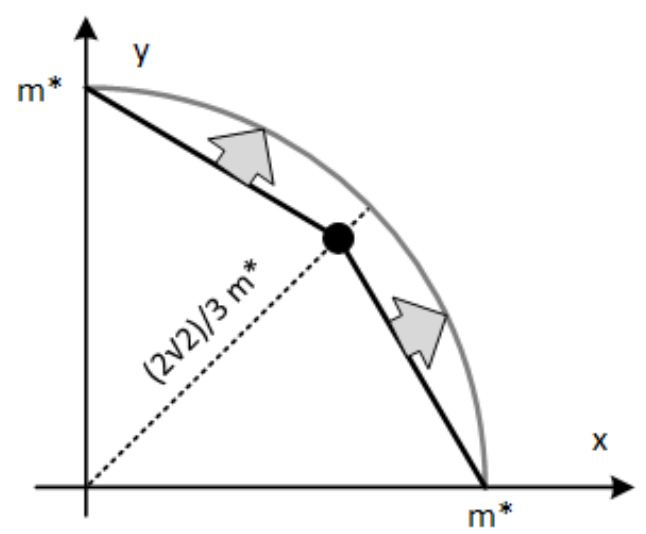

Figure 2. Segments for a computed magnitude of $m^{*}$.

If the $\mathrm{x}$ and $\mathrm{y}$ data paths are quantized on the $\mathrm{M}$ bit signed, the magnitude estimator in Figure 1 needs $\mathrm{M}$ bit unsigned to represent $m^{*}$. The idea is to segment the input space, based on $m^{*}$. We label as M1 area the set of points with the MSB (Most Significant Bit) of $m^{*}$, say $m^{*}$ [M-1], equal to one. If a point is not in the M1 area then we look for the $m^{*}[\mathrm{M}-2]$ bit to mark it in the M2 area, and so on, until the desired level of segmentation is reached. Figure 3 shows the first area segments of the input coordinates while Figure 4 shows a simple circuit able to extract the logical information of the segmented area as a function of $m^{*}$. 


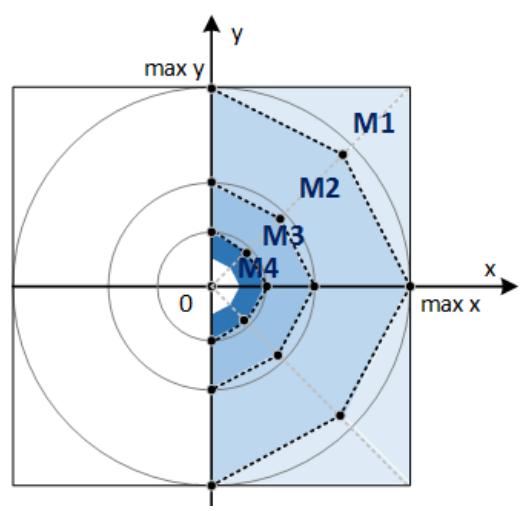

Figure 3. Segmentation of the input coordinates.

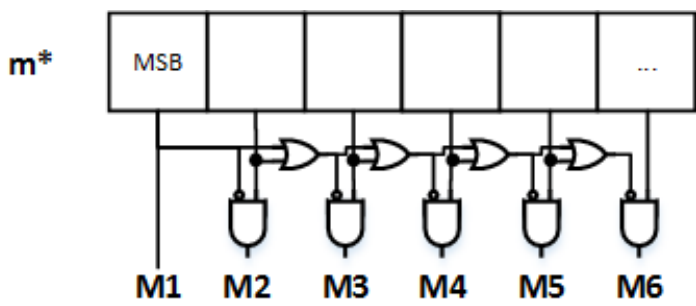

Figure 4. Segmentation flags circuit.

When the Mk area for a point is known, a shift factor (SFk) is selected and applied to the $x_{0}$ and $y_{0}$ input coordinates in a way that the magnified coordinates reach the M1 area or the M2 area. In other words, we want to work with points that are always in the first two segments, far from the zero point. Equation (4) shows the doubling rule to correctly shift the inputs starting from the M3 area (SF1 = 0, $\mathrm{SF} 2=0$ ). Figure 5 shows the architecture of the improved atan-CORDIC with the fast magnitude estimator and the input magnification. A difference compared to other CORDIC optimizations and CORDIC architectures in the literature [7-9,14-16] is the maintenance of the standard CORDIC core, to which we add a low-complexity pre-processing unit, working on the input ranges, thus minimizing the overall circuit complexity overhead.

$$
x_{0} 2^{S F_{k}} ; y_{0} 2^{S F_{k}}: S F_{k}=k-2 \quad k \geq 3
$$

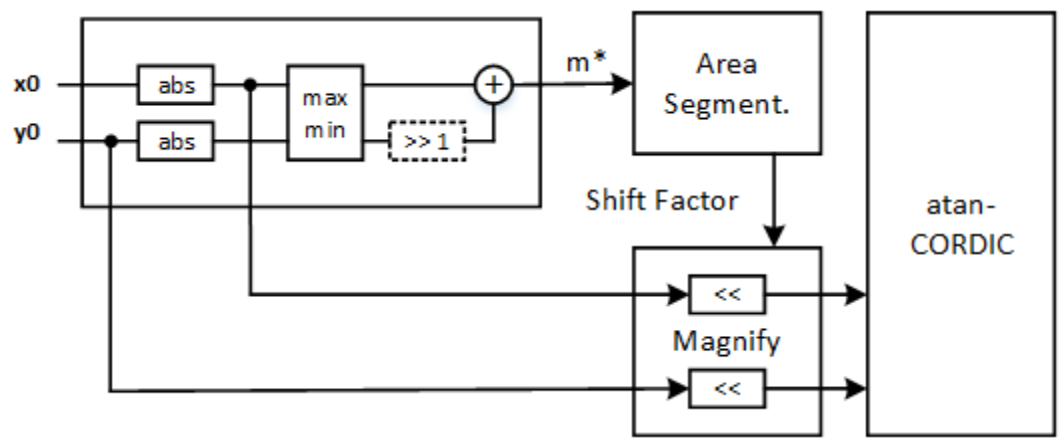

Figure 5. Improved atan-CORDIC architecture.

\section{Computation Accuracy Evaluation}

The architecture proposed in Figure 5 has been designed as a parametric HDL macrocell where the bit-width of the $x, y$ and angle data paths can be customized at synthesis time, as well as the 
number $n$ of CORDIC iterations and the number of input segments in Figure 3. For the atan-CORDIC core, a pipelined fully sequential architecture has been implemented. The choice of these parameters implies different optimization levels. An empirical parameter exploration is mandatory to characterize the sub-optimum architecture depending on the application requirements.

The quantization parameters in the fixed-point implementation are the number of bits of the $x$ and $y$ coordinates, the number of bits of the angle quantization, the number of iterations of the algorithm and the number of segmented areas. Giving the angle resolution in K bits, we know that the useful number of iterations comes from the relation where the quantized angle on the ATR set goes under half of its LSB (Last Significant Bit), being LSB $=\pi / 2^{K-1}$. Overestimating the angle $\alpha_{i}$ with $2^{-i}$, we can compute the useful limit of iterations with

$$
2^{-i}<L S B / 2 \Rightarrow i>K-\log _{2} \pi \approx K-1.65
$$

This means that the iteration index goes from 0 to $K-1$ (K iterations). As a case study, we present an atan-CORDIC with an $x, y$ data path on 14-bit signed, an angle data path on 12-bit signed and $n=12$ iterations (iteration index from 0 to 11). To understand the phase error behavior, a first analysis is done holding the $\mathrm{x}$ coordinate ( $x=16$ in the example) and sweeping the entire y space. Figure 6 shows the absolute error in radians of the computed phase with the standard CORDIC with a blue trace. We see that for points near zero, the phase error increases significantly. The max error is $\sim 0.10865 \mathrm{rad}$ (about $71 \mathrm{LSB}$ ). Using the improved atan-CORDIC with nine area segments (max pre-magnification of 128), we get the result in Figure 7. In Figure 7, the blue trace is the absolute error. In Figure 7, the max error is $\sim 0.0035 \mathrm{rad}$ (less than $3 \mathrm{LSB}$ ). The same analysis can be extended through the $\mathrm{x}$ coordinates, collecting the maximum error of each run. Figure 8 shows the standard atan-CORDIC phase errors with a blue trace where the maximum is $\sim 0.6355 \mathrm{rad}$ (about $414 \mathrm{LSB}$ ). Figure 9 shows the improved atan-CORDIC errors with a blue trace where the maximum is $\sim 0.0052 \mathrm{rad}$ (less than $4 \mathrm{LSB}$ ). The red line in Figures 6-9 is the coarse upper-bound error evaluated with Equation (2).

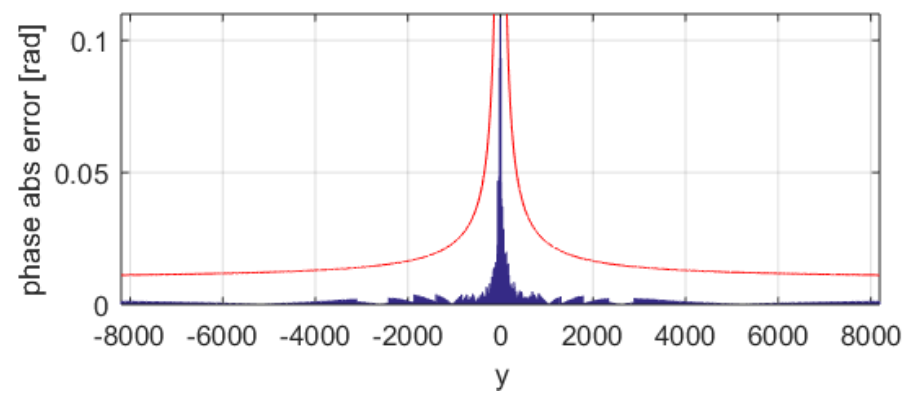

Figure 6. Standard atan-CORDIC phase error of $x=16$ and $y$. The red line is the coarse upper-bound error evaluated with Equation (2); the blue trace is the standard atan-CORDIC phase error.

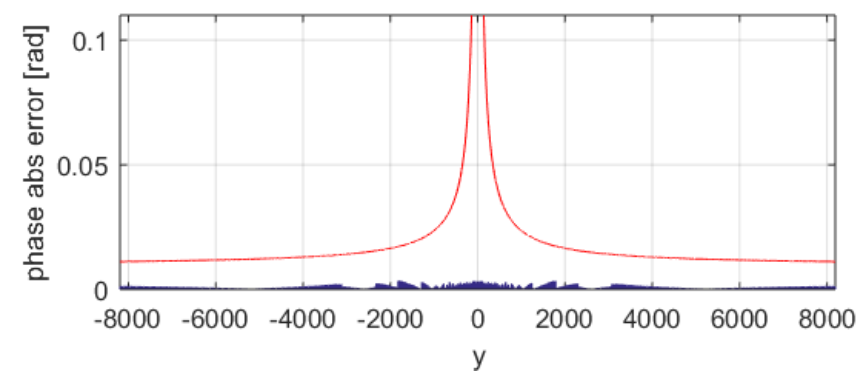

Figure 7. Improved atan-CORDIC phase error of $x=16$ and $y$. The red line is the coarse upper-bound error evaluated with Equation (2); the blue trace is the standard atan-CORDIC phase error. 


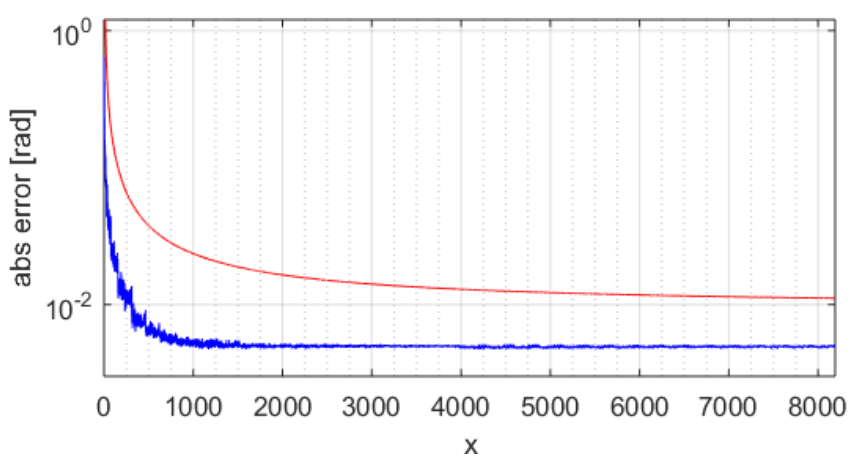

Figure 8. Standard atan-CORDIC max phase error on all the input space. The red line is the coarse upper-bound error evaluated with Equation (2); the blue trace is the standard atan-CORDIC phase error.

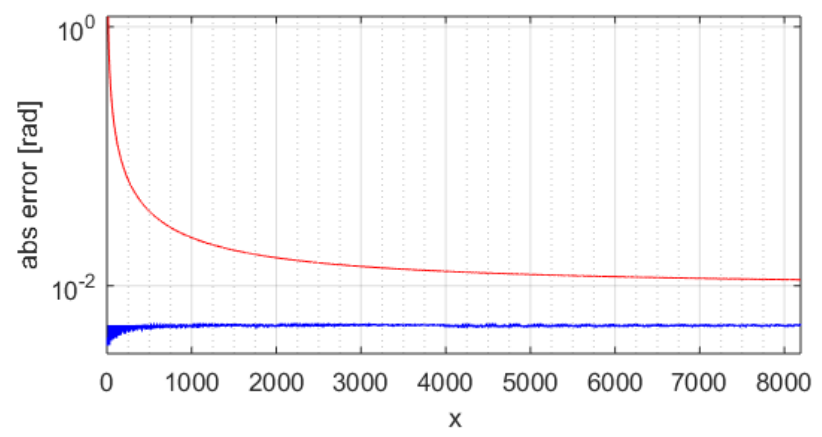

Figure 9. Improved atan-CORDIC max phase error on all the input space. The red line is the coarse upper-bound error evaluated with Equation (2); the blue trace is the standard atan-CORDIC phase error.

To evaluate further the performance of the proposed architecture, the maximum error on the angle is analyzed by varying some quantization parameters, as reported in Tables 1 and 2. In Table 1 the light cells show the standard CORDIC maximum error, fixed to 414 LSB (410 LSB in Table 2), while the grey cells represent the improved CORDIC maximum angle error. From the analysis of Tables 1 and 2, it is clear that increasing the number of $M$ areas and/or increasing the bit width of the $x$ and $y$ data paths allows reducing the error. Instead, if we compare the peak error as a function of the number of iterations $n$ (12 iterations in Table 1 vs. nine iterations in Table 2), the minimum is obtained for Table 2, which uses a number of iterations lower than the maximum 12 estimated from Equation (5), with $K=12$. This can be justified by the fact that, as discussed in Section 1, the quantization in the data paths of the fixed-point implementation causes a truncation error that is accumulated in every loop of the algorithm.

Table 1. Max angle error expressed in LSB of the standard (std) vs. the proposed (our) CORDIC, $K=12$, 12 iterations from 0 to 11, bits for $x$ and $y$ from 10 to $14, \mathrm{M}$ areas from 6 to 9 .

\begin{tabular}{cccccccccc}
\hline & \multicolumn{8}{c}{ M Areas } \\
\cline { 2 - 10 } & \multicolumn{2}{c}{$\mathbf{6}$} & \multicolumn{2}{c}{$\mathbf{7}$} & \multicolumn{2}{c}{$\mathbf{8}$} & \multicolumn{2}{c}{$\mathbf{9}$} \\
\hline $\boldsymbol{x}, \boldsymbol{y}$ Bits & std & our & std & our & std & our & std & our \\
\hline $\mathbf{1 0}$ & 414 & 50 & 414 & 16 & 414 & 16 & 414 & 16 \\
$\mathbf{1 1}$ & 414 & 50 & 414 & 13 & 414 & 10 & 414 & 10 \\
$\mathbf{1 2}$ & 414 & 50 & 414 & 13 & 414 & 10 & 414 & 6 \\
$\mathbf{1 3}$ & 414 & 50 & 414 & 13 & 414 & 10 & 414 & 4 \\
$\mathbf{1 4}$ & 414 & 50 & 414 & 13 & 414 & 10 & 414 & 4 \\
\hline
\end{tabular}


Table 2. Max angle error expressed in LSB of the standard (std) vs. the proposed (our) CORDIC, $K=12$, nine iterations from 0 to 8 , bits for $\mathrm{x}$ and $\mathrm{y}$ from 10 to 14 , M-areas from 6 to 9 .

\begin{tabular}{ccccccccc}
\hline & \multicolumn{8}{c}{ M areas } \\
\cline { 2 - 10 } & \multicolumn{2}{c}{$\mathbf{6}$} & \multicolumn{2}{c}{$\mathbf{7}$} & \multicolumn{2}{c}{$\mathbf{8}$} & \multicolumn{2}{c}{$\mathbf{9}$} \\
\hline $\boldsymbol{x}, \boldsymbol{y}$ Bits & std & our & std & our & std & our & std & our \\
\hline $\mathbf{1 0}$ & 410 & 45 & 410 & 15 & 410 & 15 & 410 & 15 \\
$\mathbf{1 1}$ & 410 & 45 & 410 & 10 & 410 & 10 & 410 & 10 \\
$\mathbf{1 2}$ & 410 & 45 & 410 & 9 & 410 & 9 & 410 & 8 \\
$\mathbf{1 3}$ & 410 & 45 & 410 & 9 & 410 & 9 & 410 & 7 \\
$\mathbf{1 4}$ & 410 & 45 & 410 & 9 & 410 & 9 & 410 & 7 \\
\hline
\end{tabular}

Indeed, in Table 3 we have reported, for both the standard and the proposed CORDIC, the maximum angle error, expressed in LSB, as a function of the number of iterations. For example, the minimum of the maximum angle error is achieved for the proposed CORDIC with seven iterations. This behavior can be theoretically justified, since the maximum angle error is achieved for low magnitude values, i.e., low values of the radius $r$ in Equation (2). Figure 10 plots the error upper bound as a function of the number of iterations, using Equation (2) with $\epsilon=1, \delta=\pi / 2^{K-1}, K=12$, and values for the radius $r$ of 8, 16, 32 and 64. In Figure 10, similarly to what is observed in Table 3 , there is a first part where the upper-bound error decreases and a second part where the upper-bound error increases.

Table 3. Max angle error expressed in LSB of the standard vs. proposed CORDIC $(K=12,12$ bits for $x$ and $y$ data paths, six $M$ areas).

\begin{tabular}{lcccccccccccc}
\hline & \multicolumn{10}{c}{ Max. Number of Iterations } \\
\cline { 2 - 14 } & $\mathbf{2}$ & $\mathbf{3}$ & $\mathbf{4}$ & $\mathbf{5}$ & $\mathbf{6}$ & $\mathbf{7}$ & $\mathbf{8}$ & $\mathbf{9}$ & $\mathbf{1 0}$ & $\mathbf{1 1}$ & $\mathbf{1 2}$ \\
\hline Standard CORDIC & 512 & 302 & 252 & 333 & 374 & 394 & 404 & 409 & 412 & 413 & 414 \\
Proposed CORDIC & 512 & 302 & 160 & 82 & 43 & 30 & 40 & 45 & 48 & 49 & 50 \\
\hline
\end{tabular}

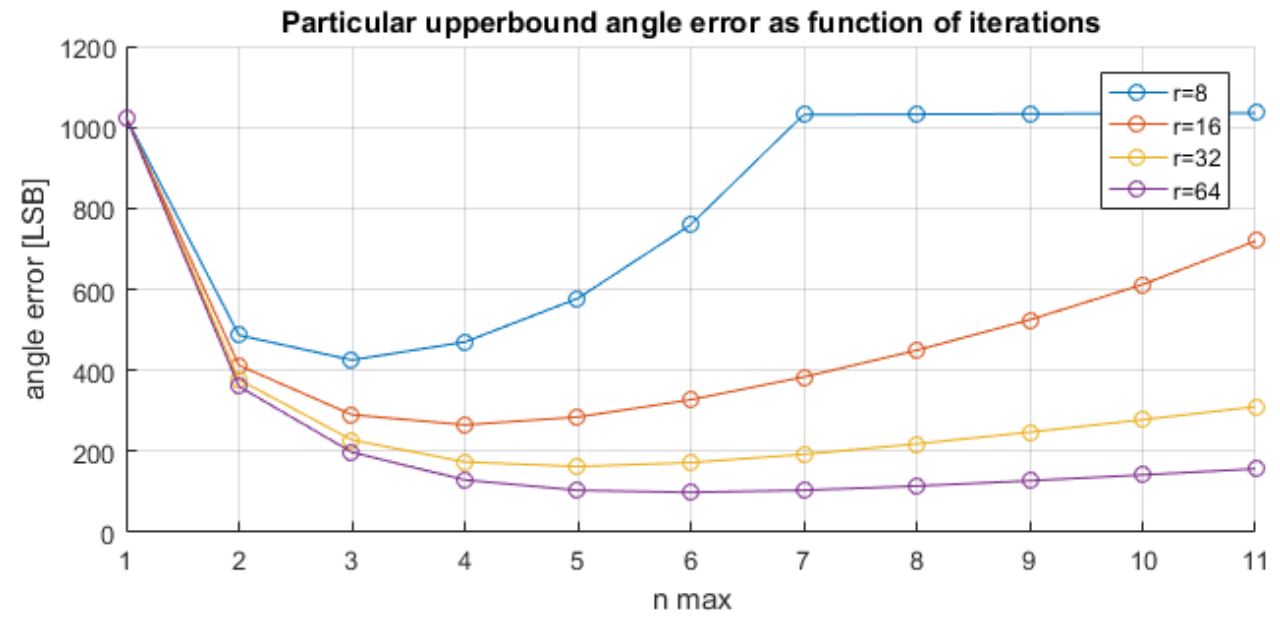

Figure 10. Upper-bound error behavior as function of the iteration number.

\section{VLSI Implementation and Characterization}

The improved CORDIC architecture proposed in Section 2 has been designed in VHDL as a parametric macrocell that can be integrated in any system-on-chip. With reference to the macrocell configuration discussed in Section 3, a synthesis in the standard-cell $180 \mathrm{~nm} 1.8 \mathrm{~V}$ CMOS technology from AMS ag has been carried out. The macrocell design is characterized by a low circuit complexity 
and low power consumption: about 1700 equivalent logic gates, with a max power consumption (static and dynamic power) of about $0.45 \mathrm{~mW}$ when working with a clock frequency of $30 \mathrm{MHz}$. The overhead due to the innovative parts of this work (fast magnitude estimation and input coordinates magnification) vs. a standard CORDIC implementation in the same technology is limited to $24 \%$ in terms of equivalent logic gates and $-36 \%$ in terms of max working frequency. The processing time of the atan function is $n$.Tclock, i.e., $0.4 \mu$ s when $n=12$ with a clock of $30 \mathrm{MHz}$. As a possible improvement, a pipeline register can be inserted in Figure 5 before the atan-CORDIC core. This way, the complexity increases by about $10 \%$, resulting in 1900 equivalent logic gates, while the working frequency can be increased up to $46 \mathrm{MHz}$ and the power consumption is $0.77 \mathrm{~mW}$. The processing time is $(n+1)$. Tclock; with $n=12$ iterations of the atan function, the processing time is $0.28 \mu \mathrm{s}$.

The comparison of the proposed solution vs. the standard state-of-the-art solution, implemented in the same $180 \mathrm{~nm} 1.8 \mathrm{~V}$ technology, is summarized in Table 4 . Table 4 shows also the comparison with other known CORDIC computation architectures [6,8]. In [8], an embedded solution is proposed using a 32-bit ART7TDMI-S core (18,000 equivalent gates) plus $32 \mathrm{kB}$ of RAM (Random Access Memory) and $512 \mathrm{kB}$ of EEPROM (Electrically Erasable Programmable Read Only Memory). With a clock of $60 \mathrm{MHz}$, the solution in [8] implements an atan evaluation in $35 \mu \mathrm{s}$ and an angle error of $2.42 \times 10^{-5} \mathrm{rad}$ with an optimized algorithm. At $60 \mathrm{MHz}$ with $180 \mathrm{~nm} 1.8 \mathrm{~V} \mathrm{CMOS}$ technology, the ARM7-TDMIs has a power consumption of about $24 \mathrm{~mW}$. Although [8] is optimized to achieve a lower error, $2.42 \times 10^{-5}$ rad vs. $6.14 \times 10^{3} \mathrm{rad}$ in our implementation, with $K=12$, the proposed design outperforms the design in [8] in terms of the reduced processing time, by a factor $\times 124$, the reduced power consumption, by a factor $\times 31$, and the reduced circuit complexity, by a factor $\times 9.5$. To achieve a similar calculation accuracy as in [8], our IP has been re-synthetized with $K=20$. In such a case, the processing time is still within $1 \mu \mathrm{s}$ and the complexity in the logic gates is still below 4 kgates.

Table 4. Performance of the proposed CORDIC solution vs. standard solution.

\begin{tabular}{ccccc}
\hline IP macrocell & Standard CORDIC & This work & [6] & [8] \\
\hline Equivalent gates & 1400 & $1700(1900$ pipelined $)$ & 8000 & $18,000+32 \mathrm{kB} \mathrm{RAM}$ \\
+ & & $30(46$ pipelined $)$ & 10 & 60 \\
Clock frequency, $\mathrm{MHz}$ & 46 & $6.1 \times 10^{-3} \mathrm{rad}$ & $\mathrm{N} / \mathrm{A}(\mathrm{K}=16, n=20)$ & $2.42 \times 10^{-5} \mathrm{rad}$ \\
Max. angle error & $0.6355 \mathrm{rad}$ & $0.4(0.28$ pipelined $)$ & $\mathrm{N} / \mathrm{A}$ & 35 \\
Processing latency, $\mu \mathrm{s}$ & 0.28 & $0.45(0.77$ pipelined $)$ & N/A & 24 \\
Power consumption, $\mathrm{mW}$ & 0.57 & & \\
\hline
\end{tabular}

The $180 \mathrm{~nm}$ technology node and the target clock frequency have been selected following the constraints of automotive applications where, to face a harsh operating environment (over-voltage, over-current, temperature up to $150{ }^{\circ} \mathrm{C}$ ), a $180 \mathrm{~nm} 1.8 \mathrm{~V}$ technology ensures higher robustness vs. scaled sub-100 nm CMOS technologies operating with a voltage supply of $1 \mathrm{~V}$. The considered $180 \mathrm{~nm}$ technology is automotive-qualified and has a logic gate density of $118 \mathrm{kgates} / \mathrm{mm}^{2}$ [18]. The N-MOS supplied at $1.8 \mathrm{~V}$ has a voltage threshold of $0.35 \mathrm{mV}$ and a current capability of $600 \mu \mathrm{A} / \mu \mathrm{m}^{2}$. The P-MOS supplied at $1.8 \mathrm{~V}$ has a voltage threshold of $0.42 \mathrm{mV}$ and a current capability of $260 \mu \mathrm{A} / \mu \mathrm{m}^{2}$.

The proposed improved CORDIC IP has been integrated in an application-specific processor, called SENSASIP [5], to improve the digital signal processing capability of a 32-bit CORTEX-M0 core. Particularly, the SENSASIP chip is used to implement the algorithms in $[3,19-21]$ in real time for the sensor signal processing of a 3D Hall sensor and of an inductive position sensor in X-by-wire automotive applications. In these specific applications, the real-time application requirements were met already with a clock frequency for SENSASIP of $8 \mathrm{MHz}$. Hence, with a $46 \mathrm{MHz}$ clock frequency, the improved CORDIC architecture is about six times faster than required. At $8 \mathrm{MHz}$, the power consumption of the proposed CORDIC architecture is below $0.1 \mathrm{~mW}$.

It should be noted that the CORDIC computation can be done via software on the 32-bit Cortex core, as is done in [15] for the same class of magnetic sensor position signal conditioning. However, as proved in [5], the use of the proposed CORDIC macrocell integrated on-chip allows for savings in 
power consumption up to $75 \%$. It should be noted that the proposed macrocell can be also integrated in FPGA technology, as in [16], in case the application of interest requires an FPGA implementation rather than a system-on-chip one.

\section{Conclusions}

In this work, we propose an improved architecture for arctangent computation using the CORDIC algorithm. A fast magnitude estimator works with a pre-processing magnification to rescale the input coordinates to an optimum range as a function of the estimated magnitude. After the scaling, the inputs are processed by the standard CORDIC algorithm implemented without changing any data path precision. The improved architecture is generic and can be applied to any atan-CORDIC implementation. The architecture improvement is achieved with a low-circuit complexity since it does not require any special operation, but only comparison, shift and add operations. Several case studies have been analyzed, considering a resolution for $x$ and $y$ from 10 to 14 bits, a resolution of 12 bits for the angle, a number of iterations from two to 12, and from six to nine area segments. The bit-true analysis shows that the maximum absolute error in the atan-CORDIC computation improves by at least two orders of magnitude, e.g., in Table 1 from 414 LSB to 4 LSB. The proposed macrocell has been integrated in a system-on-chip, called SENSASIP, for automotive sensor signal processing. When implemented in an automotive-qualified $180 \mathrm{~nm} 1.8 \mathrm{~V}$ CMOS technology, two configurations are available. The first one has a circuit complexity of 1700 logic gates, a max clock frequency of $30 \mathrm{MHz}$, a CORDIC processing time of $0.4 \mu$ s and a power consumption of $0.45 \mathrm{~mW}$. The second configuration has an extra pipeline stage, and has a circuit complexity of 1900 logic gates, a max clock frequency of $46 \mathrm{MHz}$, a CORDIC processing time of $0.28 \mu$ s and a power consumption of $0.77 \mathrm{~mW}$. These values are compared well to state-of-the-art works in the same $180 \mathrm{~nm}$ technology node. The $46 \mathrm{MHz}$ clock value is six times higher than what is required $(8 \mathrm{MHz})$ by real-time automotive sensor signal conditioning applications. In these operating conditions, the power consumption of the proposed solution is below $0.1 \mathrm{~mW}$.

Acknowledgments: This project was partially supported by the projects FP7 EU Athenis3D and the University of Pisa PRA E-TEAM.

Author Contributions: All authors contributed to the research activity and to the manuscript writing and review. Conflicts of Interest: The authors declare no conflict of interest.

\section{References}

1. Volder, J.E. The CORDIC Trigonometric Computing Technique. IRE Trans. Electr. Comput. 1959, EC-8, 330-334. [CrossRef]

2. Meher, P.K.; Valls, J.; Juang, T.B.; Sridharan, K.; Maharatna, K. 50 Years of CORDIC: Algorithms, Architectures, and Applications. IEEE Trans. Circuit Syst. I 2009, 56, 1893-1907. [CrossRef]

3. Sisto, A.; Pilato, L.; Serventi, R.; Fanucci, L. A design platform for flexible programmable DSP for automotive sensor conditioning. In Proceedings of the Nordic Circuits and Systems Conference (NORCAS): NORCHIP \& International Symposium on System-on-Chip (SoC), Oslo, Norway, 26-28 October 2015; pp. 1-4.

4. Zheng, D.; Zhang, S.; Zhang, Y.; Fan, C. Application of CORDIC in capacitive rotary encoder signal demodulation. In Proceedings of the 2012 8th IEEE International Symposium on Instrumentation and Control Technology (ISICT), London, UK, 11-13 July 2012; pp. 61-65.

5. Sisto, A.; Pilato, L.; Serventi, R.; Saponara, S.; Fanucci, L. Application specific instruction set processor for sensor conditioning in automotive applications. Microprocess. Microsyst. 2016, 47, 375-384. [CrossRef]

6. Timmermann, D.; Hahn, H.; Hosticka, B.; Schmidt, G. A Programmable CORDIC Chip for Digital Signal Processing Applications. IEEE J. Solid-State Circuits 1991, 26, 1317-1321. [CrossRef]

7. Rajan, S.; Wang, S.; Inkol, R.; Joyal, A. Efficient approximation for the arctangent function. IEEE Signal Process. Mag. 2006, 23, 108-111. [CrossRef] 
8. Ukil, A.; Shah, V.H.; Deck, B. Fast computation of arctangent functions for embedded applications: A comparative analysis. In Proceedings of the 2011 IEEE International Symposium on Industrial Electronics (ISIE), Gdansk, Poland, 27-30 June 2011; pp. 1206-1211.

9. Revathi, P.; Rao, M.V.N.; Locharla, G.R. Architecture design and FPGA implementation of CORDIC algorithm for fingerprint recognition applications. Procedia Technol. 2012, 6, 371-378. [CrossRef]

10. Walther, J.S. A unified algorithm for elementary functions. In Proceedings of the Spring Joint Computer Conference AFIPS ‘71 (Spring), Atlantic City, NJ, USA, 18-20 May 1971; pp. 379-385.

11. Hu, Y.H. The quantization effects of the CORDIC algorithm. IEEE Trans. Signal Process. 1992, 40, 834-844. [CrossRef]

12. Kota, K.; Cavallaro, J.R. A normalization scheme to reduce numerical errors in inverse tangent computations on a fixed-point CORDIC processor. In Proceedings of the 1992 IEEE International Symposium on Circuits and Systems, San Diego, CA, USA, 10-13 May 1992; Volume 1, pp. 244-247.

13. Fanucci, L.; Rovini, M. A Low-Complexity and High-Resolution Algorithm for the Magnitude Approximation of Complex Numbers. IEICE Trans. Fundam. Electron. Commun. Comput. Sci. 2002, E85-A, 1766-1769.

14. Muller, J.-M. The CORDIC Algorithm. In Elementary Functions; Birkhäuser Boston: Cambridge, MA, USA, 2016; pp. 165-184.

15. Glascott-Jones, A. Optimising Efficiency using Hardware Co-Processing for the CORDIC Algorithm. In Advanced Microsystems for Automotive Applications; Springer: Heidelberg, Germany, 2010; pp. 325-335.

16. Taylor, A.P. How to use the CORDIC algorithm in your FPGA design. XCell J. 2012, 79, 50-55.

17. Anoop, C.V.; Betta, C. Comparative Study of Fixed-Point and Floating-Point Code for a Fixed-Point Micro. In Proceedings of the dSPACE User Conference 2012, Bangalore, India, 14 September 2012.

18. Minixhofer, R.; Feilchenfeld, N.; Knaipp, M.; Röhrer, G.; Park, J.M.; Zierak, M.; Enichlmair, H.; Levy, M.; Loeffler, B.; Hershberger, D.; et al. A 120 V 180 nm High Voltage CMOS smart power technology for System-on-chip integration. In Proceedings of the 2010 22nd International Symposium on Power Semiconductor Devices \& IC's (ISPSD), Hiroshima, Japan, 6-10 June 2010.

19. Bretschneider, J.; Wilde, A.; Schneider, P.; Hohe, H.-P.; Koehler, U. Design of multidimensional magnetic position sensor systems based on hallinone technology. IEEE Int. Symp. Ind. Electron. (ISIE) 2010, $422-427$. [CrossRef]

20. Sarti, L.; Sisto, A.; Pilato, L.; di Piro, L.; Fanucci, L. Platform based design of 3D Hall sensor conditioning electronics. In Proceedings of the 2015 11th Conference on IEEE Conference on Ph.D. Research in Micr. and Electronics (PRIME), Glasgow, UK, 29 June-2 July 2015; pp. 200-203.

21. Kim, S.; Abbasizadeh, H.; Ali, I.; Kim, H.; Cho, S.; Pu, Y.; Yoo, S.-S.; Lee, M.; Hwang, K.C.; Yang, Y.; et al. An Inductive 2-D Position Detection IC With 99.8\% Accuracy for Automotive EMR Gear Control System. IEEE Trans. Very Large Scale Integr. (VLSI) Syst. 2017. [CrossRef] 